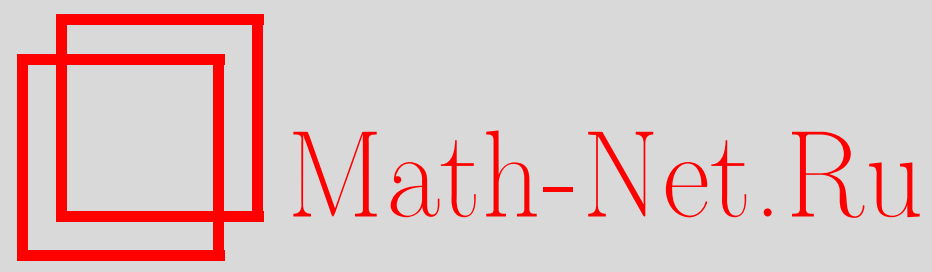

М. Г. Шур, Об условии Лина в сильных предельных теоремах для отношений, Матем. заметки, 2004, том 75, выпуск 6, 927-940

DOI: https://doi.org/10.4213/mzm77

Использование Общероссийского математического портала Math-Net.Ru подразумевает, что вы прочитали и согласны с пользовательским соглашением http://www . mathnet.ru/rus/agreement

Параметры загрузки:

IP: 52.87 .193 .239

26 апреля 2023 г., 13:20:54 


\title{
ОБ УСЛОВИИ ЛИНА В СИЛЬНЫХ ПРЕДЕЛЬНЫХ ТЕОРЕМАХ ДЛЯ ОТНОШЕНИЙ
}

М.Г. Шур

\begin{abstract}
Изложена новая версия сильных предельных теорем для отношений Лина (1976 г.), освобожденная от прежних ограничений типа абсолютной непрерьвности рассматриваемых мер или консервативности соответствующих операторов. Как показьвает наиболее оригинальное утверждение работы (см. теорему 5), одно из двух основных условий Лина выполнено автоматически для шерокого класса цепей Маркова и, в частности, для многих случайных блужданий на группах. Доказательство этого базируется на существенном развитии результатов автора (1980г.), касающихся поведения многошаговых переходных вероятностей.
\end{abstract}

Библиографоия: 13 названий.

1. Введение. Применительно к цепям Маркова сильные предельные теоремы для отношений предлагают при определенных условиях информацию о поведении отношений вида $\mu\left(P^{n} f\right) / \nu\left(P^{n} g\right)$ при $n \rightarrow \infty$. Здесь $P$ - переходный оператор какой-либо (однородной) цепи Маркова, реализованной на измеримом пространстве $(E, \mathscr{B}), f$ и $g-$ неотрицательные $\mathscr{B}$-измеримые функции, $\mu$ и $\nu$ - вероятностные меры на $\mathscr{B}$, а под $\mu(\varphi)$ понимается интеграл $\int \varphi d \mu$, если функция $\varphi \mathscr{B}$-измерима и интеграл имеет смысл. Как правило, либо при доказательстве, либо при применении упомянутых теорем приходится проверять, что $\nu\left(P^{n+1} f\right) / \nu\left(P^{n} f\right)$ стремится при $n \rightarrow \infty$ к некоторому пределу $\rho>0$, желательно не зависящему от конкретного выбора $f$ из того или иного класса, причем наибольшее внимание уделяется случаю $\rho=1$ (см., например, [1]-[5]).

Подобная проверка часто наталкивается на значительные трудности, и поэтому интересен подход Лина [6], позволяющий в ряде случаев ограничиться выводом лишь неравенства

$$
\varliminf_{n \rightarrow \infty} \frac{\nu\left(P^{n+1} f\right)}{\nu\left(P^{n} f\right)} \geqslant 1,
$$

назьваемого далее условием Лина, для подходящих $f$ и $\nu$ (строго говоря, в [6] используется вариант (1) с оператором, двойственным в определенном смысле для нашего $P$; при этом непосредственным объектом анализа в [6] служат неотрицательные сжимающие операторы). Это условие или его варианты обеспечиваются предположениями любой из известных сильных предельных теорем для отношений (см., например, [1]-[6]), исключая лишь теорему 7.5 из [2], в которой его отсутствие нам оправдать не удалось.

Работа выполнена при поддержке Российского фонда фундаментальных исследований, грант № 02-01-997. 
В п. 3 предлагаемой работы дается новая версия основных результатов из [6] (см. теоремы 1 и 3), исключающая предположения из [6], связанные с абсолютной непрерьвностью тех или иных мер или консервативностью некоторых операторов; при этом прежнее требование перемешивания заменено соответствующими положениями законов нуля-двойки Дерьеника [7], [8]. Простая теорема 2 довольно близка к упоминавшейся теореме из [2], но в отличие от нее не содержит условий типа возвратности по Харрису, зато предполагает наличие цепи, двойственной исходной. В теоремах 1-3 постулированы условия типа (1), тогда как теорема 5 из п. 5 , служащая главным результатом работы, устанавливает автоматическое вьполнение условия Лина в изрядном классе случаев, включая широкий набор случайных блужданий на группах. Ослабленньй вариант теоремы 5 и притом с неполным обоснованием см. в [9]. Доказательство этой теоремы потребовало существенной переработки (см. п. 4) результатов из [10], касающихся поведения величин вида $\left(P^{n} f\right)^{1 / n}$ и им подобных при $n \rightarrow \infty$; близкие утверждения для дискретных цепей Маркова см. в [11].

2. Основные обозначения и терминология. В основу нашей терминологии положена традиционная терминология для общей теории цепей Маркова [1], [8] с дополнениями из [2], касающимися неприводимых цепей. Раз и навсегда фиксируем измеримое пространство $(E, \mathscr{B})$ с $\sigma$-алгеброй $\mathscr{B}$, содержащей все одноточечные множества в $E$. Символом $b \mathscr{B}$ (соответственно $b \mathscr{B}_{+}$) обозначим совокупность всех ограниченных $\mathscr{B}$-измеримьг (к тому же неотрицательных) функций, а символом $\mathscr{M}_{1}-$ совокупность всех вероятностных мер на $\mathscr{B}$. Рассмотрим цепь Маркова $X=\left(X_{n} ; n \geqslant 0\right)$ с пространством состояний $(E, \mathscr{B})$ и марковской переходной вероятностью $p(x, A), x \in X, A \in \mathscr{B}$, порождающей переходньй оператор $P$ (таким образом, $P f(x)=\int f(y) p(x, d y), x \in E$, для ограниченных снизу $\mathscr{B}$-измеримых функций $f$ ). Для тех же $f$ при $\alpha \geqslant 0$ положим

$$
G^{\alpha} f=\sum_{n \geqslant 0} \alpha^{n} P^{n} f
$$

и $G f=G^{1} f$. Вводится также обозначение

$$
\mu P^{n}(A)=\int p_{n}(x, A) \mu(d x), \quad A \in \mathscr{B},
$$

где $p_{n}(\cdot, \cdot)$ - переходная вероятность цепи $X$ за $n \geqslant 1$ шагов, а $\mu$-произвольньй ограниченньй (вещественный) заряд на $\mathscr{B}$. В роли же $\|\mu\|$ будет браться соответствующая полная вариация, т.е. $\sup \{\mu(f) ; f \in b \mathscr{B}$ и $|f| \leqslant 1\}$.

Пусть $\sigma$-конечная мера $\eta$ задана на $\mathscr{B}$ и нетривиальна (т.е. $\eta \not \equiv 0)$. Функцию $f \in b \mathscr{B}_{+}$ назовем $\eta$-малой, если $\eta(f)>0$ и $G^{\alpha} f>0$ при некотором (а потому и всех) $\alpha>0$ и если для любой функции $g \in b \mathscr{B}+c \eta(g)>0$ найдутся такие $a>0$ и целое $m \geqslant 0$, что

$$
f \leqslant a P^{m} g
$$

(условия вида (3) включены в определения малых функций нескольких типов, ср. [6], [12]). Множество $A \in \mathscr{B} \eta$-мало, если $\eta$-мал его индикатор $1_{A}$. Вместе с каждой $\eta$-малой $f$ таковьпи являются и $P^{m} f, m \geqslant 1$, с $\eta\left(P^{m} f\right)>0$, и $f_{1} \in b \mathscr{B}+$ с $\eta\left(f_{1}\right)>0$, если $f_{1} \leqslant f$. Например, положительность $G^{\alpha} f_{1}$ следует из неравенства $(3)$ с $g=f_{1}$, в силу которого $G^{\alpha} f_{1} \geqslant a^{-1} \sum_{n \geqslant m} \alpha^{n} P^{n-m} f=a^{-1} \alpha^{m} G^{\alpha} f, \alpha>0$. 
Существование $\eta$-малых функций гарантировано при широких предположениях, включающих, в частности, условие минорирования Харриса [12]. Вот пример, связанньй с понятием $C$-множества в смысле [1]: пусть $A \in \mathscr{B}$ таково, что $\eta(A)>0$ и $G^{\alpha} 1_{A}>0$, и пусть $p_{m}(x, B) \geqslant b \eta(B)$ при всех $x \in A, B \in \mathscr{B}$ и некоторых $b>0$ и $m \geqslant 1$; тогда $A \eta_{1}$-мало, где $\eta_{1}(B)=\eta(A \cap B), B \in \mathscr{B}$, так как $P^{m} g \geqslant b \eta_{1}(g)$ на $A$ для $g \in b \mathscr{B}_{1}$. Цепь $X$ назовем $\eta$-апериодической, если хотя бы для одной $\eta$-малой функции $f$ (а следовательно, и для всех таких $f$ ) множество $E_{x}=\left\{n \geqslant 0: P^{n} f(x)=0\right\}$ конечно при любом $x \in E$.

Пусть на $\mathscr{B}$ задана нетривиальная $\sigma$-конечная мера $\xi$, и пусть цепь $X \xi$-неприводима $[1],[2]$, т.е.

$$
H(\cdot) \equiv \sum_{n \geqslant 1} P_{n}(\cdot, A)>0 \quad \text { в } E \quad \text { для всех } A \in \mathscr{B} \quad \text { с } \quad \xi(A)>0 .
$$

При этом, заменив при необходимости исходную меру $\xi$ другой, мы можем и будем дополнительно требовать, чтобы $H_{A}=0 \quad \xi$-почти всюду, если $\xi(A)=0[2]$. Функция $f \in b \mathscr{B}+$ считается малой (для $X$ ), если $\xi(f)>0$ и найдутся $b>0, m \geqslant 1$ и нетривиальная мера $\varkappa$ на $\mathscr{B}$ такие, что $p(\cdot, A) \geqslant b \varkappa(A) f(\cdot)$ на $E$ при всех $A \in \mathscr{B}$ (в отечественной литературе такие функции именуют также минорантными). Если $\mathscr{B}$ счетнопорождена, то, во-первых, $E$ представимо в виде счетного объединения малых множеств; во-вторых, всякая $\xi$-малая функция мала и наоборот; в-третьих, для $X$ можно указать параметр сходимости $R$, т.е. число $R \geqslant 1$ со свойствами:

a) $G^{\alpha} f<\infty$ на некотором поглощающем множестве $K$, если $0 \leqslant \alpha<R$ и функция $f$ мала;

б) $G^{\alpha} f \equiv \infty$, если $\alpha>R, f \in b \mathscr{B}+$ и $\xi(f)>0[2]$.

Напомним, что множество $A \in \mathscr{B}$ назьвается поглощающим, если на нем $p(\cdot, A)=1$.

3. Сильные предельные теоремы для отношений. Всюду в дальнейшем рассматривается цеп Маркова $X=\left(X_{n} ; n \geqslant 0\right)$ с пространством состояний $(E, \mathscr{B})$ и прочими атрибутами, указанными в п. 2 , причем часто используется

УСловиЕ (I). $\sigma$-алгебра $\mathscr{B}$ счетнопорождена, а цепь $X$ является $\xi$-неприводимой и апериодической и имеет параметр сходимости $R=1$, где $\xi$ - мера с соответствуюшими свойствами (см. п. 2).

В этом пункте дается наша версия основной части теории, представленной в [6], и до условия (IV) мы фиксируем $f \in b \mathscr{B}+$ и $\nu \in \mathscr{M}_{1}$, предположив, что $\nu\left(P^{n} f\right)>0$ при всех натуральных $n$, начиная с какого-то $n=n_{0}$. На $\mu \in \mathscr{M}_{1}$ часто налагается

УСловие (II). Имеет место сходимость $\left\|(\mu-\nu) P^{n}\right\| \rightarrow 0$ при $n \rightarrow \infty$.

Выполнение этого требования сразу для всех $\mu \in \mathscr{M}_{1}$ мы трактуем как проявление благоприятной возможности во втором законе нуля-двойки [8; гл. 6], и такая возможность наверняка осуществляется, если имеет место условие (I) и цеп $X$ возвратна по Харрису [1], [2], [8]. Если же действует первый закон нуля-двойки, то требование (II) реализуется с $\mu=\nu P^{m}, m \geqslant 1$.

Введем еще одно предположение. 
УСЛовИЕ (III). Семейство функций $\left\{f_{n} ; n \geqslant n_{0}\right\}$, где $f_{n}=P^{n} f / \nu\left(P^{n} f\right)$, равномерно ограничено в $E$.

Требования такого типа включаются во многие сильные предельные теоремы для отношений (см. [1], [2], [6], [8]).

С функцией $f$ свяжем семейство $\mathscr{S}=\mathscr{S}(f)$ всех $\varphi \in b \mathscr{B}+$, для каждой из которых найдутся такие $a, b>0$ и целые $i, j, k \geqslant 0$, что

$$
a P^{i} f \leqslant P^{j} \varphi \leqslant b P^{k} f
$$

Образуем также конус $\mathscr{K}=\mathscr{K}(f)$ из всевозможных линейных комбинаций

$$
c_{1} \varphi_{1}+\cdots+c_{n} \varphi_{n}, \quad \text { где } n \geqslant 1, \quad \varphi_{i} \in \mathscr{S}, c_{i} \geqslant 0, \quad 1 \leqslant i \leqslant n
$$

исключая случай $c_{1}=\cdots=c_{n}=0$. При условии $(\mathrm{I})$ семейства $\mathscr{S}$ и $\mathscr{K}$ содержат все малые функции, если мала $f$, а при условии (Ia) из п. 4 то же можно сказать и о $\eta$-малых функциях.

Работая с вещественными последовательностями $\left\{a_{n} ; n \geqslant 0\right\}$ и $\left\{b_{n} ; n \geqslant 0\right\}$, будем писать, как обычно, $a_{n} \sim b_{n}$, если $b_{n} \neq 0$ при достаточно больших $n$ и $a_{n} / b_{n} \rightarrow 1$ при $n \rightarrow \infty$.

ТЕОрема 1. Пусть фиксированные выше $f$ и $\nu$ подчиняются условию (III), $а$ $\mu \in \mathscr{M}_{1}$ удовлетворяет условию (II). Если при этом реализуется (1), то

$$
\mu\left(P^{n} f\right) \sim \nu\left(P^{n} f\right)
$$

а если, кроме того, имеет место (II) с мерой $\nu P$ вместо $\mu$, то

$$
\mu\left(P^{n+1} f\right) \sim \mu\left(P^{n} f\right) \quad u \quad \nu\left(P^{n+1} f\right) \sim \nu\left(P^{n} f\right) .
$$

Более того, при выполнении всех указанных условий соотношения (5) и (6) сохранят силу, если в них $f$ заменить любой функиией $\varphi \in \mathscr{K}$ (в частности, любой малой $\varphi$, коль скоро действует (I) $u f$ мала).

ДоКАЗАТЕЛЬСТво (ср. [6]). Положим

$$
J_{n}(\varphi)=\left|\mu\left(P^{n} \varphi\right)-\nu\left(P^{n} \varphi\right)\right| \quad \text { для } \varphi \in b \mathscr{B}_{+} \cdot
$$

Пусть вьполнены условия, предшествующие (5), и, следовательно, $\left|f_{n}\right| \leqslant M$ при некотором $M>0$ и всех $n \geqslant n_{0}$. Задав $m \geqslant 1$ и считая $n$ достаточно большим, получаем

$$
J_{n}(f)=\left|(\mu-\nu)\left(P^{m}\left(P^{n-m} f\right)\right)\right|=\left|(\mu-\nu) P^{m}\left(f_{n-m}\right)\right| \nu\left(P^{n-m} f\right),
$$

благодаря чему

$$
J_{n}(f) \leqslant 2 M\left\|(\mu-\nu) P^{m}\right\| \nu\left(P^{n} f\right)
$$

в соответствии с определением нормы заряда и неравенством $\nu\left(P^{n-m} f\right) \leqslant 2 \nu\left(P^{n} f\right)$, вытекающим из (1). Приведенная оценка для $J_{n}(f)$ и условие (II) влекут (5). Если выполняется еще и (II) с $\nu P$ в роли $\mu$, то применимо (5) с $\nu P$ в той же роли. Таким образом, второе, а вместе с ним и первое соотношения в (6) обоснованы. 
Докажем заключительную часть теоремы. Пусть $\varphi \in \mathscr{S}$, т.е. выполнено (4) с подходящими $a, b, i$ и т.д. Считая снова $n$ достаточно большим, имеем $P^{n} \varphi=P^{q} P^{j} \varphi$ с $q=n-j$, а также

$$
\begin{aligned}
P^{n} \varphi & \leqslant b P^{q+k} f=b f_{q+k} \nu\left(P^{q+k} f\right), \\
\nu\left(P^{n} \varphi\right) & \geqslant a \nu\left(P^{q+i} f\right) \geqslant \frac{a}{2} \nu\left(P^{N} f\right),
\end{aligned}
$$

где $N=q-m+k$, a $m \geqslant k-i$ произвольно. Отсюда подобно прежнему

$$
J_{n}(\varphi) \leqslant\left\|(\mu-\nu) P^{m}\right\| \cdot \sup _{E} P^{n-m} \varphi \leqslant b M\left\|(\mu-\nu) P^{m}\right\| \nu\left(P^{N} f\right),
$$

вследствие чего

$$
\left|J_{n}(\varphi)\right| \leqslant M_{1}\left\|(\mu-\nu) P^{m}\right\| \nu\left(P^{n} \varphi\right)
$$

при подходящем $M_{1}>0$. Это снова приводит к (5), а затем и к (6), но с $\varphi$ в роли $f$. Случай $\varphi \in \mathscr{S}$ рассмотрен, а переход к $\varphi \in \mathscr{K}$ тривиален.

ЗАмЕчАниЕ 1. Попутно было показано, что при осуществлении всех условий теоремы 1 , приведших к (5) и (6), любая функция $\varphi \in \mathscr{S}=\mathscr{S}(f)$ подчиняется требованиям типа (1) и (III).

ЗАмЕчАниЕ 2. Иногда (см. далеепример 1) вместо (III) удобнее использовать более громоздкое

УСловиЕ (IIIa). Пространство $E$ является объединением $\mathscr{B}$-измеримых множеств $E_{1} \subset E_{2} \subset \cdots$ таких, что для любого $E_{m}, m \geqslant 1$, существует $E_{n}, n \geqslant 1$, для которого $p\left(\cdot, E_{n}\right)=1$ на $E_{m} ;$ при этом семейство $\left\{f_{n} ; n \geqslant n_{0}\right\}$ (см. (III)) финально равномерно ограничено (т.е. при некотором $M>0$ и любом натуральном $m$ применимо неравенство $\left|f_{n}\right|<M$ на $E_{m}$, если $n$ превьшает некоторое натуральное $\left.N_{m}\right)$.

Теорема 1 и ее доказательство сохранят силу и при использовании (IIIa) вместо (III), коль скоро ограничиться рассмотрением мер $\mu$ и $\nu$, сосредоточенных на каком-либо $E_{m}$, $m \geqslant 1$. То же относится к теоремам 2,3 и следствию 1 .

Введем теперь

УСловиЕ (IV). Для исходной цепи $X$ и некоторой нетривиальной $\sigma$-конечной инвариантной для $X$ меры $\pi$ существует двойственная цепь Маркова $\widehat{X}$.

Таким образом, это условие требует существования цепи Маркова $\widehat{X}$ с прежним пространством состояний и переходным оператором $\widehat{P}$, для которой

$$
\pi(g P f)=\pi(f \widehat{P} g) \quad \text { для } f, g \in b \mathscr{B}_{+} \cdot
$$

Если выполнено (IV) вместе с каким-либо условием (·), относящимся к $X$, то, переформулировав $(\cdot)$ в терминах, отвечающих $\widehat{X}$, получим новое условие $(\cdot)^{d}$, называемое двойственным для $(\cdot)$. Пример: под $(1)^{d}$ понимается $(1)$ с $P$, замененным на $\widehat{P}$. Говоря же о реализации некоторого условия (скажем, (III)) для каких-либо функции $g$ и меры $\nu_{1}$, будем подразумевать его осуществление с $g$ и $\nu_{1}$ вместо исходных $f$ и $\nu$. 
TЕорема 2. Пусть реализуются как условие (IV), так $u$ (II) $u$ (II) ${ }^{d}$ для всех $\mu, \nu \in \mathscr{M}_{1}$. Пусть найдутся функиии $f, g, w \in \mathscr{B}_{+}$, суммируемые по $\pi, u$ мерь $\mu_{0}, \nu_{0}, \varkappa \in \mathscr{M}_{1}$ такие, что для обеих пар $\left(f, \mu_{0}\right) u\left(g, \nu_{0}\right)$ выполняются условия (1) $u$ (III), а для $(w, \varkappa)-$ условия (1) ${ }^{d} u$ (III) ${ }^{d}$. Тогда для всех $\mu, \nu \in \mathscr{M}_{1}$

$$
\lim _{n \rightarrow \infty} \frac{\mu\left(P^{n} f\right)}{\nu\left(P^{n} g\right)}=\frac{\pi(f)}{\pi(g)} .
$$

ДокАЗАТЕЛЬСТво проведем, считая, что $\pi(f)=\pi(g)=\pi(w)=1$, так как этого вполне достаточно. Рассмотрим меры $\mu_{1}, \nu_{1}, \varkappa_{1}$ из $\mathscr{M}_{1}$, имеющие относительно $\pi$ плотности $f, g, w$ соответственно. По теореме 1

$$
\mu\left(P^{n} f\right) \sim \mu_{0}\left(P^{n} f\right) \sim \varkappa_{1}\left(P^{n} f\right)=\pi\left(w P^{n} f\right)=\mu_{1}\left(\widehat{P}^{n} w\right) \sim \varkappa\left(\widehat{P}^{n} w\right) .
$$

Аналогично, $\nu\left(P^{n} g\right) \sim \varkappa\left(\widehat{P}^{n} w\right)$ и, следовательно, предел, указанньй в $(8)$, существует и равен 1. Тем самьм, (8) доказано в интересующем нас случае.

СлЕДСТВИЕ 1. Пусть одновременно реализуются (I) и условия из первой фразы теоремы 2. Пусть найдутся $\mu_{0}, \varkappa \in \mathscr{M}_{1}$ и малая (для $X$ ) функиия $f$ и функиия $w \in \mathscr{B}_{+}$, суммируемая по $\pi$, такие, что пара $\left(f, \mu_{0}\right)$ подчиняется условиям (1) $u$ (III), а пара $(w, \varkappa)-$ условиям (1) ${ }^{d} u$ (III) ${ }^{d}$. Тогда (8) сf $и g$, замененными на $\varphi$ и $\psi$, применимо ко всем малым функииям $\varphi$ и и всем $\mu, \nu \in \mathscr{M}_{1}$.

ДокАЗАТЕЛЬСтво. В самом деле, (I) влечет суммируемость по $\pi$ всех малых функций. По замечанию 1 условия (1) и (II) приложимы ко всем малым $\varphi$, так что можно применить теорему 2 , рассмотрев пары $\left(\varphi, \mu_{0}\right),\left(\psi, \mu_{0}\right)$ и $(w, \varkappa)$ с любыми малыми $\varphi$ и $\psi$.

Теорема 2 находит приложение при рассмотрении следующих примера 1 и теоремы 3.

ПримеР 1. Пусть $E$ является хаусдорфовой локально компактной сепарабельной группой с совокупностью $\mathscr{B}$ своих борелевских подмножеств и правой мерой Хаара $\pi$. В роли $X$ возьмем правое случайное блуждание на $E$ с законом $\zeta \in \mathscr{M}_{1}$ (т.е. $p(x, A)=$ $p\left(e, x^{-1} A\right)=\zeta\left(x^{-1} A\right), x \in E, A \in \mathscr{B}$, где $e$ - нейтральньй элемент групш, а групповая операция записьвается как умножение). Потребуем, чтобы

a) хотя бы одна сверточная степень меры $\zeta$ была несингулярна относительно $\pi$;

б) $E$ являлась единственной из своих замкнутых подгрупп, на которой сосредоточена $\zeta$, и $\zeta$ не была сосредоточена ни на одном смежном классе по какой-либо собственной замкнутой нормальной подгруппе.

Тогда (см. упражнение 3.17 из [8; гл. 3]) применимо условие (I) с возможным изъятием требования $R=1$, цепь $X$ апериодична, а всякая $f \in b \mathscr{B}+$ c $\pi(f)>0$, равная 0 , вне какого-либо компакта, является малой. Вьполняется и (IV) с теперешней мерой $\pi$ и случайным блужданием $\hat{X}$, закон $\hat{\zeta}$ которого индуцирован мерой $\zeta$ при отображении $x \rightarrow x^{-1}, x \in E$, и которое обладает свойствами, отмеченными вьше для $X$.

Потребуем дополнительно, чтобы група $E$ являлась абелевой, закон $\zeta$ имел компактньй носитель и параметр сходимости цепи $X$ равнялся 1 . Тогда $X$ и $\widehat{X}$ подчиняются условиям (II) и (II) ${ }^{d}$ соответственно [7]. Теперь легко вывести (8) для $f, g \in b \mathscr{B}_{+}$ при условии, что $f=g=0$ вне некоторого компакта, $\pi(g)>0$, а $\mu, \nu \in \mathscr{M}_{1}$ имеют компактные носители (теорема 2.4 из [6] здесь дает несколько меньше). Для этого выделим 
непустое открытое множество $B \subset E$ с компактным замыканием и положим $f=w=1_{B}$ и $\mu_{0}(A)=\varkappa(A)=\pi(A \cap B) / \pi(B), A \in \mathscr{B}$. Согласно [13] имеют место условия следствия 1 с (IIIa) и (IIIa) ${ }^{d}$ вместо (III) и (III) ${ }^{d}$, причем множества $E_{n}$ из (IIIa) и (IIIa) ${ }^{d}$ можно считать компактными. Замечание 2 и следствие 1 влекут (8) в интересующей нас сейчас ситуации.

При условии (IV) цепь $X$ называется симметричной относительно $\pi$, если $P=\widehat{P}$.

СлЕДСТВИЕ 2. Пусть выполнено (IV), иепь X симметрична относительно $\pi u$ реализуется (II) для всех $\mu, \nu \in \mathscr{M}_{1}$. Если найдутся функиии $f, g \in$ b̆̊̈ + , суммируемые по $\pi$, и меры $\mu_{0}, \nu_{0} \in \mathscr{M}_{1}$ такие, что обе пары $\left(f, \mu_{0}\right) u\left(g, \nu_{0}\right)$ удовлетворяют условиям (1) $u$ (III), то при любых $\mu, \nu \in \mathscr{M}_{1}$ применимо (8).

Здесь достаточно сослаться на теорему 2 , взяв $w=f$ и $\varkappa=\mu_{0}$.

Пусть цепь Маркова $X$ симметрична относительно $\pi$, и пусть $\mathscr{L}^{2}=\left\{f \in b \mathscr{B}: \pi\left(f^{2}\right)\right.$ $<\infty\}$. Для $f, g \in \mathscr{L}^{2}$ положим $(f, g)=\pi(f g)$ и $\|f\|=(f, f)^{1 / 2}$. Известно, что для $f \in \mathscr{L}^{2} \cap b \mathscr{B}_{+}$с $\pi(f)>0$ сушествует предел $\rho(f)=\lim _{n \rightarrow \infty}\left(\left\|P^{n+1} f\right\| /\left\|P^{n} f\right\|\right)($ см. [6]; более простое доказательство см. в [5]); при $\pi(f)=0$ положим $\rho(f)=0$. Случаем $\rho(f)<1$ заниматься не будем (при условии (I) он малоинтересен).

ТЕОрема 3. Пусть иепь $X$ подчиняется (IV), симметрична относительно $\pi и$ удовлетворяет (II) при всех $\mu, \nu \in \mathscr{M}_{1}$. Пусть $f \in \mathscr{L}^{2} \cap b \mathscr{B}_{+}, \rho(f)=1$ и осуществляется частныи й случай требования (III): функиии $P^{2 n} f /\left\|P^{n} f\right\|^{2}=P^{2 n} f /\left(P^{2 n} f, f\right)$, $n \geqslant 1$, равномерно ограничены. Пусть, наконеи, функиия $g \in b \mathscr{B}+c \pi(g)>0$ такова, что $g \leqslant M\left(f+P f+\cdots+P^{m} f\right)$ при некоторых $\mathscr{M}>0 u m \geqslant 1$. Тогда $п р и$ всех $\mu, \nu \in \mathscr{M}_{1}$ применимо равенство (8).

ДокАЗАТЕЛЬСтво. Как и ранее, ограничимся случаем $\pi(f)=\pi(g)=1$. Так как $\rho(f)=1$, то $\left(P^{2 n+2} f, f\right) \sim\left(P^{2 n} f, f\right)$. Поэтому к цепи Маркова $\left\{X_{2 n} ; n \geqslant 0\right\}$, функции $f$ и мере $\nu_{1}$ с плотностью $d \nu_{1} / d \pi=f$ применима теорема 1 , ввиду которой $\mu\left(P^{2 n} f\right) \sim$ $\nu\left(P^{2 n} f\right)$ для $\mu, \nu \in \mathscr{M}_{1}$. Записав последнее соотношение с $\mu P$ вместо $\mu$, получаем $\mu\left(P^{n+1} f\right) \sim \mu\left(P^{n} f\right) \sim \nu\left(P^{n} f\right)$. Теперь условия теоремы влекут равномерную ограниченность функций $P^{n} f /\left(P^{n} f, f\right)$ при $n \geqslant n_{1}$ (под $n_{1}$ и $n_{2}$ подразумеваются подходящие постоянные) и, кроме того, $\left(f, P^{n} g\right)=\left(g, P^{n} f\right) \sim\left(g, P^{n+1} f\right) \sim\left(f, P^{n+1} f\right)$ при $i \geqslant 1$ и $\left(f, P^{n+1} g\right) \sim\left(f, P^{n} g\right)$. Учитьвая сказанное и свойства $g$, находим

$$
\varphi_{n} \equiv \frac{P^{n} g}{\left(f, P^{n} g\right)} \leqslant M_{1} \sum_{0 \leqslant i \leqslant m} \frac{P^{n+i} f}{\left(f, P^{n+i} f\right)}, \quad \text { если } M_{1}>M, n \geqslant n_{2} \text {, }
$$

т.е. получаем равномерную ограниченность функций $\varphi_{n}, n \geqslant n_{2}$. Итак, каждая из пар $\left(g, \nu_{1}\right)$ и $\left(f, \nu_{1}\right)$ удовлетворяет условиям (1) и (III), что вместе со следствием 2 влечет $(8)$.

СлЕДСТВИЕ 3 (ср. следствие 1). Пусть выполнены условия, включенные в первые две фразы теоремы 3, и условие (I). Если функиия $f$ мала, то применимо (8) $c$ любыми мальми $g$ и $\mu, \nu \in \mathscr{M}_{1}$.

Отметим, что в этой же ситуации $f \in \mathscr{L}^{2}$, коль скоро $f$ мала.

4. Возврат к работе [10]. Этот пункт является подготовительным к п. 5, согласно которому условие Лина (1), неоднократно появлявшееся вьше, вьполняется в широком 
классе случаев. Основной интерес в п. 4 представляют теорема 4 и отчасти лемма 2 , частные случаи которых, касаюшиеся лишь неприводимых цепей и $C$-множеств, содержатся в [10].

Начнем с обсуждения следующего требования.

УСловиЕ (Іа). Пересечение любых поглощающих множеств $A_{1} \supset A_{2} \supset \cdots$ непусто (и, следовательно, само является поглощающим множеством).

Это условие вытекает как из (I), так и из существования на $\mathscr{B}$ такой конечной меры $\varkappa$, что $\varkappa(A)>\gamma$ для некоторого $\gamma>0$ и всех поглощающих $A$. Еще одно достаточное условие для (Іа) приведено в [4].

ПРЕДЛОЖЕНИЕ 1. Пусть выполнено (Ia), и пусть некоторое непустое семейство $W \subset$ в $\mathscr{B}_{+}$обладает свойством: если $f \in W$, то $G^{\alpha} f>0$ при некотором ( значит, и любом) $\alpha>0$, а если ещ, и $g \in W$, то при подходящих $а>0$ и $\mathrm{m} \geqslant 0$ (зависящих от $f$ и $g$ ) справедливо (3). Тогда существуют параметр сходимости для $W$, т.е. постоянная $\mathscr{R} \in[1, \infty)$ и поглощающее $K \subset E$ такие, что

a) $G^{\alpha} f<\infty$ на $K$, если $0 \leqslant \alpha<\mathscr{R} u f \in W$,

б) $G^{\alpha} f \equiv \infty$, если $\alpha>R u f \in W$.

ДокаЗАтЕльство. Задав $f \in W$, можно считать, что $f \leqslant a P^{m} f$ с $a \geqslant 1$ и некоторьм $m \geqslant 1$. Поэтому $P^{n} f \leqslant a P^{n+m} f, n \geqslant 0$, и последовательность чисел $\left\{a^{n m+i} \times\right.$ $\left.P^{n m+i} f\left(x_{0}\right) ; n \geqslant 0\right\}$, где $x_{0} \in E$ и $i \geqslant 0$ фиксированы, не убьвает. Поскольку $G^{\alpha} f>0$, при подходящем $i$ все ее элементы, начиная с некоторого, превьшают некое $b>0$. Следовательно, $G^{a} f\left(x_{0}\right)=\infty$, и варьирование $x_{0}$ дает тождество $G^{a} f \equiv \infty$, которое ввиду (3) распространяется с исходной функции $f$ на все $f \in W$.

Положим

$$
\mathscr{R}=\inf \left\{\alpha>0: G^{\alpha} f \equiv \infty\right\}
$$

для какой-либо $f \in W$. Ясно, что это определение не зависит от конкретного выбора $f$ и что $\mathscr{R} \geqslant 1$. Пусть числа $a_{n} \in[0, \mathscr{R})$, не убьвая, сходятся к $\mathscr{R}$ при $n \rightarrow \infty$. Множества

$$
K_{n}=\left\{x \in E: G^{a_{n}} f(x)<\infty\right\}, \quad n \geqslant 1,
$$

являются поглощаюшими и $K_{1} \supset K_{2} \supset \cdots$, так что пара $\mathscr{R}$ и $K=\bigcap_{n \geqslant 1} K_{n}$ является искомой.

С этого момента фиксируем нетривиальную $\sigma$-конечную меру $\eta$ на $\mathscr{B}$ и предположим, что семейство $W(\eta)$ всех $\eta$-малых функций непусто. В дальнейшем, применяя (Ia), в качестве семейства $W$ из предложения 1 будем брать $W(\eta)$. Под $R(\eta)$ будет пониматься параметр сходимости для $W(\eta)$, а под $K(\eta)$ - соответствующее множество $K$ из предложения 1. Если цепь $X$-неприводима, то $R(\xi)$ совпадает с ее параметром сходимости $R$, а $K(\eta)$ - с множеством $K$ из определения $R$ (см. п. 2).

Отметим, что в следствиях 1 и 3 , как и в заключительной части теоремы 1 , можно было бы говорить об условии (Ia) и $\eta$-малых функциях вместо (I) и малых функций.

Лемма 1. Пусть выполнено (Іа) и заданы $\eta$-малая функиия $f$ и числа $s>R(\eta) u$ $M>0$. Тогда при некотором натуральном $r_{1}=r_{1}(s, M)$ и любых натуральных $n$ всюду в $E$

$$
\sum_{1 \leqslant m \leqslant r_{1}} s^{n+m} P^{n+m} f \geqslant s^{n} M P^{n} f .
$$


ДокАЗАТЕльство. По предложению 1 сумма $S_{n}=\sum_{1 \leqslant m \leqslant n} s^{m} P^{m} f$ стремится к $\infty$ всюду в $E$, когда $n \rightarrow \infty$, а по теореме Егорова это происходит равномерно на некотором множестве $B$ с $\eta(B)>0$. Кроме того, согласно определению $\eta$-малой функции имеем $p_{i}(\cdot, B) \geqslant a f(\cdot)$ при некоторых $i \geqslant 1$ и $a>0$, откуда $p_{n+i}(\cdot, B) \geqslant a P^{n} f(\cdot)$, $n \geqslant 1$. Поэтому, положив $M_{1}=M / a$, получаем $S_{N}>M_{1}$ на $B$ при некотором $N \geqslant 1$. Остается заметить, что при $r_{1}=N+i$

$$
\begin{gathered}
\sum_{1 \leqslant m \leqslant r_{1}} s^{n+m} P^{n+m} f(\cdot) \geqslant \sum_{1 \leqslant m \leqslant N} s^{n+m+i} \int_{B} P^{m} f(y) p_{n+i}(\cdot, d y) \\
\geqslant s^{n+i} \int_{B} S_{N}(y) p_{n+i}(\cdot, d y) \geqslant s^{n} M_{1} p_{n+i}(\cdot, B) \geqslant s^{n} M P^{n} f(\cdot) .
\end{gathered}
$$

ЛЕмма 2. Пусть выполнено (Ia) $u u_{n}=s^{n} P^{n} f, n \geqslant 1$, ф фиксированными $s>R(\eta)$ и $\eta$-малой функиией $f$. Тогда найдется такое натуральное $r=r(s)$, что для любого $x \in E$ мохсно подобрать натуральные $n_{j}=n_{j}(x), j \geqslant 1$, с соблюдением двух требований: $0<n_{j+1}-n_{j} \leqslant r u$

$$
u_{n_{j+1}}(x) \geqslant u_{n_{j}}(x), \quad j \geqslant 1 .
$$

ДокАЗАТЕльСтво. Выберем $\alpha \in(0,1)$ так, чтобы $s^{\prime}=\alpha s$ превысило $R(\eta)$, положим $M=\alpha+\alpha^{2}+\cdots$ и, следуя лемме 1 , рассмотрим $r_{1}=r_{1}(s, M)$. Ясно, что всюду в $E$

$$
\sum_{1 \leqslant m \leqslant r_{1}} u_{n+m}(x) \geqslant M u_{n}(x), \quad n \geqslant 1 .
$$

Задав $x \in E$, искомые $n_{j}=n_{j}(x)$ определим индуктивно. Пусть $n_{1}=1$. Если $n_{k}$, $1 \leqslant k \leqslant j$, уже определены, среди чисел $u_{i}=u_{i}(x)$ с $i \in\left(n_{j}, n_{j}+r_{1}\right]$ можно найти хотя бы одно, для которого $u_{i} \geqslant \alpha^{i-n_{j}} u_{n_{j}}$ (в противном случае $\sum_{1 \leqslant m \leqslant r_{1}} u_{n_{j}+m}<$ $\left(\alpha+\alpha^{2}+\cdots\right) u_{n_{j}}$, что противоречит (11)). Мы приравниваем $n_{j+1}$ к номеру первого из таких $u_{i}$ и получаем

$$
u_{n_{j+1}} \geqslant \alpha^{n_{j+1}-n_{j}} u_{n_{j}}, \quad \text { т.e. } \quad s_{1}^{n_{j+1}} P^{n_{j+1}} \geqslant s_{1}^{n_{j}} P^{n_{j}} f(x)
$$

c $s_{1}=\alpha^{-1} s$, причем пока условие $\alpha s>R(\eta)$ не использовалось. Поэтому, заменив $s$ на $s^{\prime}=\alpha s$ и затем переопределив соответствующим образом $r_{1}$ и $n_{j}$, вновь получим (12), но с $s_{1}=\alpha^{-1} s^{\prime}=s$. Тем самым (10) доказано, а за $r$ надо взять новое $r_{1}$.

Сохранив условия и обозначения из леммы 2, имеем

СлЕДСТВИЕ 4. Каковы бы ни были $x \in E$ и набор из подряд идущих $r_{2}=2 r$ натуральных чисел, неравенство $u_{l+1}(x) \geqslant u_{l}(x)$ реализуется хотя бы для одного әлемента $l=l(x)$ этого набора.

В самом деле, если это не так, то найдутся $x \in E$ и набор указанного вида такие, что $u_{n}(x)>u_{n+1}(x)$ для всех $n$ из этого набора. Но в нем содержится новый набор, составленный из чисел $n \in\left[n_{j}, n_{j+1}\right)$ при некотором $j \geqslant 1$, где $n_{j}$ то же, что и в лемме 2, и как следствие $u_{n_{j}}(x)>u_{n_{j+1}}(x)$. Это противоречит (10) и, тем самым, влечет требуемое.

В следующей теореме используются обозначения $R(\eta)$ и $K(\eta)$, введенные после предложения 1. 
Теорема 4. Пусть реализуется условие (Ia), цепь Маркова X $\eta$-апериодична и существует хотя бы одна $\eta$-малая функиия. Если $f \in b \mathscr{B}+u \eta(G f)>0$, то всюду в $E$

$$
\varliminf_{n \rightarrow \infty}\left[\left(P^{n} f\right)^{1 / n}\right] \geqslant R(\eta)^{-1},
$$

а если $f$ п-мала, то на мнохестве $K(\eta)$

$$
\lim _{n \rightarrow \infty}\left[\left(P^{n} f\right)^{1 / n}\right]=R(\eta)^{-1}
$$

При $R(\eta)=1$ последнее равенство выполняется на всем $E$ для всех $f \in b_{+} c$ $\eta(G f)>0$.

ДокАЗАТЕЛЬСТво. Пусть сначала $R(\eta) \geqslant 1$ произвольно, а $f \eta$-мала. Вследствие $\eta$-апериодичности $X$ объединение множеств

$$
E_{n}=\bigcap_{m \geqslant n}\left\{x \in E: P^{m} f(x)>0\right\}, \quad n \geqslant 1,
$$

совпадает с $E$. Поэтому $\eta\left(E_{N}\right)>0$ при каком-то $N>0$. Заданному $s>R(\eta)$ сопоставим число $r$ из леммы 2 и положим

$$
g=\min \left\{P^{m} f ; m \in[N, N+r]\right\} .
$$

Ввиду неравенства $\eta(g)>0$ имеем $P^{k} g \geqslant b f$ при подходящих $k \geqslant 0$ и $b>0$, так что $P^{k+m} f \geqslant P^{k} g \geqslant b f$ при $m \in[N, N+r]$. Задав $x \in E$ и следуя лемме 2, рассмотрим $n_{j}=n_{j}(x), j \geqslant 1$, и зафиксируем $i \geqslant 1$, при котором $P^{n_{i}} f(x)>0$. Неравенство $n_{j+1}-n_{j} \leqslant r, j \geqslant 1$, позволяет для любого $n>n_{i}+k+r+N$ подобрать $n_{j}$ так, чтобы $m=n-k-n_{j} \in[N, N+r]$. Тогда $n_{j} \geqslant n_{i}$ и в силу ранее сказанного $P^{n-n_{j}} f=P^{k+m} f \geqslant b f$, что вместе с (10) дает

$$
P^{n} f(x)=P^{n_{j}} P^{n-n_{j}} f(x) \geqslant b P^{n_{j}} f(x) \geqslant b s^{n_{i}-n_{j}} P^{n_{i}} f(x) .
$$

Таким образом, левая часть (13) оказывается не меньше величины

$$
\varliminf_{n \rightarrow \infty}\left[\left(s^{n_{i}-n_{j}}\right)^{1 / n}\right]=s^{-1},
$$

где последнее равенство вытекает из представления $n_{i}-n_{j}=m_{n}-n$, в котором $m_{n}=n-n_{j}+n_{i}$ ограничено. Выбирая исходное $s$ сколь угодно близким к $R(\eta)$, приходим к $(13)$, но пока лишь для $\eta$-малых $f$.

Если $f \in b \mathscr{B}+$ и $\eta(G f)>0$, то $\eta\left(P^{l} f\right)>0$ при некотором $l \geqslant 0$ и, какова бы ни была $\eta$-малая функция $f_{1}$, имеем $P^{m+l} f \geqslant a f_{1}$ при подходяших $m$ и $a>0$. Этот факт ввиду возможности использовать (13) применительно к $f_{1}$ приводит к (13) в требуемой общности.

Пусть снова $f \quad \eta$-мала. По своему определению величина $R(\eta)$ при $x \in K(\eta)$ равна радиусу сходимости степенного ряда $\sum_{n \geqslant 1} t^{n} P^{n} f(x), t>0$, откуда на основании классической формулы Коши-Адамара для радиуса сходимости получаем

$$
R(\eta)^{-1}=\varlimsup_{n \rightarrow \infty}\left\{\left[P^{n} f(x)\right]^{1 / n}\right\} \quad \text { для } x \in K(\eta) .
$$

Вместе с (13) это дает (14).

Заключительное утверждение теоремы вытекает из (13), поскольку только что указанный верхний предел не превьшает 1 для $f \in b \mathscr{B}_{+}$. 
СЛЕДСТВИЕ 5. Пусть выполнены условия из первой фразы теоремы $4 u R(\eta)=1$. Если $\nu \in \mathscr{M}_{1}$, a $f \in \mathscr{B}_{+}$такова, что $\eta(G f)>0$, то

$$
\left[\nu\left(P^{n} f\right)\right]^{1 / n} \rightarrow 1 \quad \text { npu } \quad n \rightarrow \infty .
$$

ДокАЗАТЕЛЬСтво. Действительно, при любом $t \in(0,1)$ множества

$$
B_{n}=\bigcap_{m \geqslant n}\left\{x \in E: P^{m} f(x)>t^{m}\right\}
$$

с ростом $n \geqslant 1$ не убьвают и согласно (13) их объединение совпадает с $E$. Таким обра3ом,

$$
C \geqslant \nu\left(P^{n} f\right) \geqslant t^{n} \nu\left(B_{n}\right) \geqslant \frac{1}{2} t^{n} \nu(E)
$$

при достаточно больших $n$ и подходящем $C>0$, откуда

$$
\varliminf_{n \rightarrow \infty}\left\{\left[\nu\left(P^{n} f\right)\right]^{1 / n}\right\} \geqslant 1
$$

что дает требуемое (ср. конец предыдущего доказательства).

ЗАмЕчАниЕ 3. Легко переформулировать леммы 1,2 и теорему 4 при условии (I) с исключенным из него требованием $R=1$, заменив $\eta$ на $\xi, R(\eta)$ на $R$ и т.д. (ср. следствия 1 и 3 ).

5. Проверка условия Лина. Здесь одна из основных ролей отведена следующему требованию.

УсловиЕ (V). Существуют неотрищательные ядра

$$
q_{n}(x, A), \quad n \geqslant 1, \quad x \in E, \quad A \in \mathscr{B},
$$

невырождаюшиеся в том смысле, что

$$
\inf _{E} q_{n}(\cdot, E)>0, \quad n \geqslant 1
$$

и такие, что

$$
p_{j}(\cdot, \cdot) \geqslant q_{n}(\cdot, \cdot), \quad 1 \leqslant j \leqslant n .
$$

Назвав в $(\mathrm{V})$ функции $q_{n}(\cdot, \cdot)$ ядрами, мы подразумеваем их $\mathscr{B}$-измеримость по первому аргументу и счетную аддитивность по второму [2], [8]. Если в (V) при некотором натуральном $N$ неравенства $n \geqslant 1$ и $1 \leqslant j \leqslant n$ заменить на $n \geqslant N$ и $N \leqslant j \leqslant n$, то возникнет ослабленное условие, которое назовем условием (Va) (см. далее пример 1a).

Теорема 5. Пусть реализуются условия (Ia) $u(\mathrm{~V})$, и пусть иепь Маркова $X$ $\eta$-апериодична и множество ее $\eta$-мальх функиий непусто. $E с л и R(\eta)=1$, то для любих $f \in b \mathscr{B}+c \eta(G f)>0 u \nu \in \mathscr{M}_{1}$ справедливо равенство

$$
\varliminf_{n \rightarrow \infty} \frac{\nu\left(P^{n+1} f\right)}{\nu\left(P^{n} f\right)}=1 .
$$


ДоКАЗАТЕЛЬСТВо. Цепь $X$ удобно считать построенной каноническим образом на каноническом выборочном пространстве $\Omega$ [2], [8]. Пусть $\mathrm{P}$ (соответственно, $\mathrm{P}_{\boldsymbol{x}}$ ) - вероятностная мера в $\Omega$, отвечающая $X$ при начальном распределении $\nu$ (при начальном состоянии $x \in E$ ). Будем использовать обозначение $\mathrm{E}\{\chi ; \Lambda\}=\int_{\Lambda} \chi d \mathrm{P}$, если указанньй интеграл имеет смысл для случайной величины $\chi$ и случайного события $\Lambda$. Аналогичный смысл имеет $\mathrm{E}_{x}\{\chi ; \Lambda\}$.

Задав $\varepsilon \in(0,1)$ и натуральное $n$, рассмотрим момент остановки $\tau_{n}$, равный наименьшему $m=0,1, \ldots, n$, при котором

$$
P^{n-m+1} f\left(X_{m}\right) \geqslant(1-\varepsilon) P^{n-m} f\left(X_{m}\right)
$$

(по определению, $\tau_{n}=n+1$, если таких $m$ нет). Основная часть доказательства теоремы состоит в выводе неравенств (16) и (17). В соответствии с первым из них

$$
\mathrm{E}\left\{f\left(X_{n}\right) ; \tau_{n} \leqslant n\right\} \leqslant(1-\varepsilon)^{-1} \mathrm{E}\left\{f\left(X_{n+1}\right) ; \tau_{n} \leqslant n\right\}
$$

что доказьвается без труда: по марковскому свойству [2], [8] левая часть (16) совпадает C

$$
\begin{aligned}
& \sum_{m=0}^{n} \mathrm{E}\left\{f\left(X_{n}\right) ; \tau_{n}=m\right\}=\sum_{m=0}^{n} \mathrm{E}\left\{P^{n-m} f\left(X_{m}\right) ; \tau_{n}=m\right\} \\
& \quad \leqslant(1-\varepsilon)^{-1} \sum_{m=0}^{n} \mathrm{E}\left\{P^{n-m+1} f\left(X_{m}\right) ; \tau_{n}=m\right\}=(1-\varepsilon)^{-1} \sum_{m=0}^{n} \mathrm{E}\left\{f\left(X_{n+1}\right) ; \tau_{n}=m\right\},
\end{aligned}
$$

а последнее выражение равно правой части (16).

Далее, применив следствие 4 в случае $s=(1-\varepsilon)^{-1}$, найдем такоенатуральное $r_{2}$, что среди любых $r_{2}$ подряд идущих натуральных чисел содержится $l=l(x)$, при котором $P^{l+1} f(x) \geqslant(1-\varepsilon) P^{l} f(x)$, и фиксируем натуральное $k>r_{2}$. Нам предстоит при $n>k$ вывести второе из недавно упоминавшихся неравенств:

$$
\mathrm{P}_{x}\left\{\tau_{n}>q\right\} \leqslant d \mathrm{P}_{x}\left\{\tau_{n}>q-k\right\}
$$

для $x \in E$ и натуральных $q \in(k, n]$, где $d=d(\varepsilon) \in(0,1)$ независит от рассматриваемых $x, n$ или $q$. Для этого положим $t=q-k$ и заметим, что

$$
\left\{\tau_{n}>q\right\}=\Omega_{1} \cap \Omega_{2}, \quad \text { где } \Omega_{1}=\left\{\tau_{n}>t\right\}, \quad \Omega_{2}=\bigcap_{t+1 \leqslant i \leqslant q}\left\{X_{i} \in B_{i}\right\}
$$

если

$$
B_{i}=\left\{x \in E: P^{n-i+1} f(x)<(1-\varepsilon) P^{n-i} f(x)\right\}
$$

Поскольку

$$
\left\{X_{i} \in B_{i}\right\}=\theta_{t}^{-1}\left\{X_{i-t} \in B_{i}\right\}, \quad i \geqslant t_{1},
$$

где $\theta$ - канонический оператор сдвига в $\Omega$ [2], [8], то $\Omega_{2}=\theta_{t}^{-1} \Lambda$, если $A_{j}=B_{j+t}$ и $\Lambda=\bigcap_{1 \leqslant j \leqslant k}\left\{X_{j} \in A_{j}\right\}$. Следовательно, вновь по марковскому свойству

$$
\mathrm{P}_{x}\left\{\tau_{n}>q\right\}=\mathrm{E}_{x}\left\{\mathrm{P}_{X_{t}}\{\Lambda\} ; \tau_{n}>t\right\}, \quad x \in E,
$$


и для обоснования (17) остается найти приемлемую постоянную, ограничивающую сверху правую часть очевидного неравенства

$$
\mathrm{P}_{x}\{\Lambda\} \leqslant \min \left\{p_{j}\left(x, A_{j}\right), 1 \leqslant j \leqslant k\right\}
$$

С этой целью воспользуемся равенством $E=\bigcup_{1 \leqslant j \leqslant k}\left(E \backslash A_{j}\right)$, обусловленным выбором $r_{2}, k$ и $A_{j}$. Согласно $(\mathrm{V})$ для любого $x \in E$ при некотором $j_{0} \in[1, k]$, зависящем, как правило, от $x, n$ и $q$, справедливы соотношения

$$
q_{k}\left(x, E \backslash A_{j_{0}}\right) \geqslant k^{-1} q_{k}(x, E)>a
$$

с подходящим $a=a(\varepsilon) \in\left(0, k^{-1}\right)$, влекущие неравенство $p_{j_{0}}\left(x, E \backslash A_{j_{0}}\right)>a$. Поэтому в качестве искомой постоянной можно взять $d=1-a>0$, что ввиду (18) и (19) влечет неравенство (17).

Заставив $q$ последовательно пробегать значения $n, n-k, \ldots, n-l k$, где $l$ равно целой части числа $n / k$, вьводим из (17) оценку

$$
\mathrm{P}_{x}\left\{\tau_{n}>n\right\} \leqslant d^{n / k-1}, \quad x \in E .
$$

Следовательно, разности

$$
\nu\left(P^{n} f\right)-\mathrm{E}\left\{f\left(X_{n}\right) ; \tau_{n} \leqslant n\right\} \quad \text { и } \quad \nu\left(P^{n+1} f\right)-\mathrm{E}\left\{f\left(X_{n+1}\right) ; \tau_{n} \leqslant n\right\}
$$

при $n \rightarrow \infty$ стремятся к 0 , по меньшей мере, с геометрической скоростью, тогда как по следствию 5 величина $\nu\left(P^{n} f\right)$, если и стремится к 0 , то гораздо медленнее. Отсюда и из (16) заключаем, что каково бы ни было $\varepsilon>0$, левая часть (15) не может быть меньшей $1-\varepsilon$, и теорема доказана.

ЗАмечание 4. Теорема 5 сохранит силу, если в ней (V) заменить условием (Va), сформулированным вслед за (V). Чтобы в этом убедиться, в последнем доказательстве заменим, во-первых, условие $k>r_{2}$ на $k>r_{2}+N$ с тем же $N$, что и в (Va), и, во-вторых, неравенства $1 \leqslant j \leqslant k$ из (19) на $N \leqslant j \leqslant k$. Опираясь на равенство $E=\bigcup_{N \leqslant j \leqslant k}\left(E \backslash A_{j}\right)$, вновь получим оценку вида $q_{k}\left(x, E \backslash A_{j_{0}}\right)>a>0$, но с $j_{0} \in[N, k]$, а остальные рассуждения не требуют изменений.

ПримеР 1а. Возвратившись к началу примера 1, рассмотрим ту же (возможно, неабелеву) группу $E$ и правое случайное блуждание на ней $X$ с $R=1$ и законом, подчиненным тем же условиям а) и б). Как и ранее, ссылка на [8] гарантирует, помимо прежних свойств $X$, еще и осуществление ( Va). Отсюда и из замечания 4 вытекает (15) для $\nu \in \mathscr{M}_{1}$ и $f \in b \mathscr{B}_{+}$с $\pi(f)>0$. 


\section{СПИСОК ЦИТИРОВАННОЙ ЛИТЕРАТУРЫ}

[1] Orey S. Lecture Notes on Limit Theorems for Markov Chain Transition Probabilities. London: Van Nostrand, 1971.

[2] Нуммелин Э. Общие неприводимые цепи Маркова и неотрицательные операторы. М.: Мир, 1989.

[3] Guivarc'h Y. Théorèmes quotientes pour les marches aléatoires // Astérisque. 1980. V. 74. P. $15-28$.

[4] Шур М. Г. Асимптотические свойства степеней положительных операторов. I // Теор. вероятн. и ее примен. 1984. Т. 29. № 4. С. 692-702; II // Теор. вероятн. и ее примен. 1985. T. 30. № 2. C. 241-251.

[5] Шур М. Г. Предельные теоремы для отношений, ассоциированных с самосопряженными операторами и симметричными цепями Маркова // Теор. вероятн. и ее примен. 2000. Т. 45. № 2. C. 252-268.

[6] Lin M. Strong ratio limit theorems for mixing Markov operators // Ann. Inst. Poincaré. 1976. V. 12. № 2. P. 181-191.

[7] Derrienic Y. Lois "zero ou deux" pour les processus de Markov // Ann. Inst. Poincaré. 1976. V. 12. № 2. P. 111-129.

[8] Ревюз Д. Цепи Маркова. М.: РФФИ, 1997.

[9] Shur M. G. New ratio limit theorems for Markov chains // Analytic Methods in Applied Probability. In memory of F. Karpelevich / ed. Yu. M. Suhov. Amer. Math. Soc. Transl. (2). V. 207. Providence, RI: Amer. Math. Soc., 2002. P. 203-212.

[10] Шур М. Г. Асимптотическое поведение многошаговых вероятностей перехода // Литовский матем. ж. 1980. Т. 20. № 4. С. 201-207.

[11] Kingman J. F. C. The exponential decay of Markov transition probabilities // Proc. London Math. Soc. 1963. V. 13. № 50. P. 593-604.

[12] Horowits S. On $\sigma$-finite invariant measures for Markov processes // Israel J. Math. 1968. V. 6. № 2. P. 338-345.

[13] Stone C. Ratio limit theorems for random walks on groups // Trans. Amer. Math. Soc. 1966. V. 125. № 1. P. 86-100. 